\title{
Evaluation of Infection, Prevention and Control Program in Goromonzi District, Zimbabwe, 2018: A process outcome evaluation
}

\section{Govha Emmanuel}

University of Zimbabwe College of Health Sciences

\section{Zizhou Simukai Tirivanhu}

mashonaland East Provincial Medical Directorate MOHCC

\section{Shambira Gerald}

University of Zimbabwe College of Health Sciences

\section{Gombe Tafara Notion}

African Field Epidemiology Network

Tsitsi Juru ( $\nabla$ tsitsijuru@gmail.com )

University of Zimbabwe College of Health Sciences https://orcid.org/0000-0002-3570-2331

\section{Simbarashe Chiwanda}

University of Zimbabwe College of Health Sciences

\section{Tshimanga Mufuta}

University of Zimbabwe College of Health Sciences

\section{Research article}

Keywords: Infection, Prevention and Control, Healthcare Infections, Goromonzi District

Posted Date: December 6th, 2019

DOI: https://doi.org/10.21203/rs.2.18251/v1

License: (9) (i) This work is licensed under a Creative Commons Attribution 4.0 International License.

Read Full License 


\section{Abstract}

Background A healthcare-associated infection (HAl) is defined as an infection originating in the environment of a health facility that was not present or incubating at the time of patient admission. HAls can be prevented through infection, prevention and control (IPC) measures. No hazard identification and risk assessment IPC rounds and monthly meetings were conducted in Goromonzi district since 1st of January to 30th of June 2018. No trainings nor orientation for the new employees was conducted. We therefore evaluated Goromonzi District IPC program.

Methods A process-outcome evaluation using the logic model was conducted in Goromonzi district's 15 health facilities. Checklists, interviewer administered questionnaires and key informant guides were used to collect data on availability of inputs, knowledge of health workers, processes performed, outputs and outcomes achieved. Data were entered into Epi Info 7TM, which was used to generate frequencies and proportions. Qualitative data from checklists and key informants interviews was sorted manually into themes and analysed.

Results All 15 health facilities had adequate stocks of HIV test kits and PEP kits. Adequate bins and detergents were found in only $3 / 15(20 \%)$ of health facilities. All facilities failed to hold a single IPC meeting and none had specific budget for IPC in 2018. No IPC mentorship activities were carried out in the district. Only 7/13 (54\%) health workers who had needle pricks received PEP with 2/7 (29\%) of them finishing the course. No health facility had a functional HAl surveillance system. The overall knowledge rating was fair.

Conclusion The IPC program inputs in Goromonzi district were inadequate hence its failure to achieve the intended outputs and outcomes. Inadequate knowledge, unavailability of health worker training plans, specific budgets and absence of IPC committees reflected non prioritisation of the program.

\section{Background}

A healthcare-associated infection (HAl) is defined as an infection originating in the environment of a health facility that was not present or incubating at the time of patient admission (1). HAls are one of the most common adverse events in health care delivery (1). The most common types of HAl include infections of surgical wounds, the blood stream, the urinary tract and the lower respiratory tract (3). On average at any given time, $7 \%$ of patients in high income and $10 \%$ in low income countries will acquire at least one HAl with $10 \%$ of affected patients dying (2). Although evidence is limited on the economic burden of HAl, particularly in low to medium income countries, available data from the United States of America (USA) and Europe suggest a multi-billion dollar impact (5). A large percentage of HAl are preventable through effective infection, prevention and control (IPC) measures.

Despite evidence of loss of experienced and skilled staff in the health sector, which together with deteriorating infrastructure and resources contributes to deteriorating IPC practices in Zimbabwe, information on the prevalence of HAls is scarce (6). In 2009 the World Health Organisation (WHO) 
published guidelines on core components for IPC to provide evidence-based recommendations on the IPC programmes that should be adopted by all countries to prevent HAI and to combat anti-microbial resistance (AMR) (2). With technical support from the Centres for Disease Control (CDC) and financial support from President's Emergency Plan for AIDS Relief (PEPFAR), Zimbabwe responded to the call by WHO through the development of the new IPC program Policy in 2012 (1).

The overall goal of the IPC program in Zimbabwe is to reduce the burden of health care associated infections, through improving IPC practices in all health facilities and prevent the transmission of infectious diseases, amongst staff, patients and visitors. This goal can be met by ensuring availability of policies and guidelines for procedures to be used, presence of management structures of IPC at all health facilities and availability of resources and supplies. These IPC interventions will result in the improvement of the quality of health care workers and create safer work environments that will further contribute to the attainment of the goals of the MOHCC National Health Strategic Plan 2016-2020 (8).

Provision of Post Exposure Prophylaxis (PEP) to occupational human immunodeficiency virus (HIV) exposed health care workers is a key component of IPC. Despite presence of IPC focal person and a functional HIV/AIDS program, in Goromonzi District, only (19/59)32\% of those exposed received PEP and (4/19) $21 \%$ finished the course in 2017. According to the IPC policy all health workers should be screened for tuberculosis (TB) biannually but analysis of records in Goromonzi district only $11.5 \%$ of health worker were screened at least once for TB since $1^{\text {st }}$ of January 2017 to $31^{\text {st }}$ December 2017. The District has never conducted hazard identification and risk assessment IPC rounds and monthly meetings since $1^{\text {st }}$ of January 2018 to $30^{\text {th }}$ of June 2018. No trainings or orientation had been conducted for the new employees from $1^{\text {st }}$ of January 2017 to $30^{\text {th }}$ of June 2018. Apart from that the district hospital does not have a proper incinerator hence relying on an old septic tank to destroy infectious materials using diesel.

Some departments were improvising cardboard boxes to use as waste bins and sharps boxes. This necessitates acquisition of preventable HAls by many health care workers, patients and visitors with resultant loss of working hours, less productivity, more financial spending on health and reduced life expectancy. A rise in the infectious disease prevalence leads to retarded national economic development. Loss of skilled and experienced health care workers (HCW) results in higher replacement and training costs. We evaluated the Goromonzi District IPC program to come up with reasons for poor performance and subsequent recommendations.

\section{Methods}

We conducted a process-outcome evaluation using the logic model. A Logic Model approach was used for problem analysis (Figure 1). This was used to assess the inputs which were injected into the program, the processes carried out, the outputs realized and the outcomes of the IPC program.

The study was conducted in Goromonzi district located in Mashonaland East, one of the ten administrative provinces in Zimbabwe. Goromonzi district is a peri-urban area and according to the 2012 
census, it had a total population of 224987 . It has an unemployment rate of above $80 \%$. The main economic activities in the district include peasant faming and gold panning. Goromonzi district has 27 clinics and two hospitals namely Makumbe and Ruwa rehabilitation hospital. These health care centres are far apart hence residents are still walking for more than $10 \mathrm{~km}$ to get care. Residents of Goromonzi district depends on wells and boreholes as their sources of drinking water.

\section{Sample size calculation and sampling}

Assuming that $76.9 \%$ of health care workers washed their hands after contact with blood or body fluids (Mushonga A, 2007), at $95 \%$ confidence level, a desired precision of $10 \%$ and using the Dobson Formula $n=z^{2} p(1-p) / d^{2}$ we calculated a sample size of 68 . Factoring in $10 \%$ non-response rate, the adjusted sample size was 75 .

Goromonzi district has 27 clinics and 2 hospitals namely Makumbe and Ruwa. The two hospitals were purposively recruited into the study because of their high staff establishment and 13 clinics were randomly selected making a total of $15(>50 \%)$ facilities. On average a health facility is maned by four health care workers. Therefore for us to reach our sample size, three health workers were randomly selected from those who were on duty on the day of the interview. At hospitals, four health care workers were randomly selected from each of the following departments: male ward, female ward, maternity ward, outpatients and two from the kitchen. Matron, hospital services administrator, IPC focal person, HIV/TB focal persons and district medical officer (DMO) were purposively recruited into the study as key informants.

\section{Data collection procedure and tools}

We used an observational checklist to check for presence of waste management facilities, availability of resources, resources management strategies in place, shortages of inputs that were experienced. Records review were done to assess the output and outcome indicators in Goromonzi district IPC program. We used interviewer administered questionnaires to collect information on demographics and knowledge of health care workers on IPC. It was also used to assess for IPC processes. The questionnaire was specifically developed for this current study (Additional file 1). Pictures were taken and observations done to show availability and state of toilets, waste management and handwashing facilities and IPC practices in Goromonzi district. A key informant guide was used to collect information on plans made, budget allocations, trainings and support and supervision done from the key informants.

\section{Data processing and analysis}

The public health officer did all the questionnaires analysis and data abstraction. Data were checked for completeness and accuracy then entered into Epi info version 7, which was used to generate frequencies, means and proportions. Qualitative data from checklists and key informants interviews were checked and sorted manually into themes and then analysed. A Likert type scale was used to rate respondent's 
knowledge using ratings like good, fair and poor. Those who got less than 4 questions correct were rated as poor, those between 4 and 5 , fair and those above 5 were rated as good.

\section{Ethical Consideration and Permission}

Permission to carry out the study was obtained from the Provincial Medical Directorate Mashonaland East Province and the Health Studies Office. Ethical approval was obtained from the Medical Research Council of Zimbabwe ( MRCZ/ 2018/104). Written informed consent was obtained from study participants prior to the interviews which were held privately and information obtained was not disclosed to any persons other than those relevant for the purposes of the study. Confidentiality was assured and maintained by not recording participant names and any other individual identification information on the questionnaires.

\section{Results}

Of the targeted 75 health care workers from 15 health facilities in Goromonzi district 71 of them were interviewed giving a response rate of $94 \%$. Five key informants were recruited namely DMO, hospital services administrator, IPC focal person, HIV focal person and TB focal person.

\section{Demographic characteristics of health care workers}

Out of the 71 participants 46 (64.8\%) were females. Registered general nurses were more than a third $25 / 71(35 \%)$ of the participants. Primary care nurses contributed $15 / 71(21 \%)$ of participants and $12 / 71$ (16.9\%) were general hands. Environmental health technicians (EHTs) had only 1/71 (1.4\%) participant. The median age of the participants was 38.5 years and median time in service was 9.5 years. (Table 1 )

\section{Availability of IPC resources in the 15 health facilities}

All 15 health facilities had adequate and had never experienced stock outs in HIV test kits and PEP kits. Out of the 15 health facilities 14 (93\%) had functional autoclaving equipment and 13/15(87\%) had inadequate toilets for the patients. Out of the 15 facilities $12(80 \%)$ had adequate gloves with no stock outs recorded since January 2018 . Only $8 / 15(53 \%)$ of the facilities had adequate cleaning materials like brooms and mops. Adequate bins, bin liners and detergents were found in only $3 / 15(20 \%)$ of health facilities. None of the facilities had specific budget plans for IPC. (Table 2)

\section{Processes for the IPC program}

For the period under review, Goromonzi district had four planned IPC trainings which were not implemented since January 2018 to October 2018. Out of the 15 health facilities in Goromonzi district none of them managed to hold a single IPC meeting in 2018. No IPC mentorship activities were carried out in the district during the same period. Although all the 15 facilities reported that they received quarterly support and supervision on IPC only $6 / 15(40 \%)$ had minutes. We noted that these support and 
supervisions visits were not specific for IPC but rather a component of result based funding (RBF) supervision.

Although 49/71 (35\%) respondents reported that they held monthly IPC meetings at their facilities, no proof of minutes were available. None of the 15 facilities had ever held a HAl surveillance meeting. All the 15 facilities in Goromonzi district were providing TB infection control education to their clients as required. Examination of food handlers was being done yearly and all food handlers in the hospital kitchen had valid certificates. (Table 3)

\section{Outputs for the IPC program}

In Goromonzi district, there was no health workers trained in IPC for the period January 2018 to October 2018. Out of the 323 health workers in the district only 37 (11.5\%) were screened at least once for TB since January 2018. Out of the 13 health care workers who had needle pricks only 7 (54\%) received PEP with $2 / 7(29 \%)$ of them finishing the course.

Severe side effects of PEP was cited as the major reason for not finishing the course by $5 / 7(71 \%)$ of respondents. The district never held an IPC meeting out of the 135 (15 facilities $\times 9$ months) expected since January 2018. Only 5/15(33\%) of health facilities in Goromonzi district had isolation areas for communicable diseases. Only $1 / 15(6.7 \%)$ of the facilities had an IPC committee and this is the district hospital. Eight seven percent (13/15) of the health facilities including the two hospitals had no incinerators. Eleven (100\%) kitchen workers had valid food handler's examination certificates. (Table 4)

Knowledge of participants on IPC in Goromonzi district, 2018.

The overall knowledge rating was fair with $33 / 71(47 \%)$ answering between four and five knowledge attributes correctly and only 15/71(11\%) were rated as good. Out of 71 participants 67 (94.4\%) have ever heard of IPC and 49/71(69\%) knew its ultimate goal. Half of the participants knew how to dilute disinfectants with 34/71(47\%) knowing at least 3 standard precautions of IPC. Although 43/71 (61.4\%) were aware that waste should be segregated only $17 / 71(24 \%)$ knew the bin colour coding used for segregation. Only $23 / 71(32 \%)$ of respondents were aware that they should be screened biannually for TB and $22 / 71(31 \%)$ had been screened at least once since January 2018. (Table 5)

Outcomes of the IPC program in Goromonzi district from January 2018 to October 2018.

None of the 15 health facilities in Goromonzi district had an effective HAl surveillance system hence could not ascertain its prevalence. Apart from that only $1 / 15(6.7 \%)$ which is the district hospital had a patient waiting time monitoring system at its OI/TB clinic.

Reasons for poor performance in IPC in Goromonzi district

The majority $64 / 71$ (90.1\%) of participants cited lack of resources which include detergents, bins, bin liners, cleaning equipment and PPEs as the major drawback in the IPC program. Almost two thirds 46/71 
(65\%) mentioned inadequate knowledge with 43/71 (60.6\%) reporting inadequate support and supervision on IPC. High workload was cited by $43 / 71$ (60.6\%) and the majority were nurses from clinics. Half of the respondents $36 / 71$ (51\%) mentioned inadequate guidelines on IPC as a contributory factor to poor performance.

IPC practices in Goromonzi district, 2018

\section{Correct disposal of waste}

Out of 71 participants $28(39 \%)$ cited correct waste disposal as one of the IPC precautions. It was noted that $13 / 15(87 \%)$ of health facilities including the two hospitals were using pits as incinerators and 10/15 $(67 \%)$ had improvised cardboard boxes that were used as bins. Only $3 / 15(20 \%)$ of facilities had bin liners with only $1 / 15(6.7 \%)$ having both red and black colours.

\section{Sterilization of medical equipment}

At the time of the research all 15 facilities had sterilizing equipment with Ruwa clinic mentioning that they had no gas. At 1/15 (7\%) facility, the sterilizing equipment was being kept in the toilet. At another facility, the equipment was not being used cautiously with a broken gas stove being used for heating near paper bins. At the district hospital theatre equipment was seen outside for sun drying prior to sterilization.

\section{Prevention of needle pricks}

Out of the 71 participants 29 (41\%) reported prevention of needle pricks at workplace as an IPC standard precaution. Due to lack of incinerators $9 / 15(60 \%)$ of health facilities were burning their waste and needles in open pits and at $6 / 15(40 \%)$ of facilities, full sharps boxes and empty drug vials were seen piled either outside or inside the facilities.

\section{Hand washing with soap}

Although the majority $43 / 71$ (61\%) of participants cited hand hygiene as a standard precaution of IPC only $6 / 15(40 \%)$ of facilities had soap at their handwashing facilities. Makumbe hospital had no enough hand washing facilities in the male and female wards. Out of the 17 health care workers who were observed during consultation only seven $(41 \%)$ were seen washing their hands with soap after examining or dressing a patient.

\section{Discussion}

In our evaluation of the IPC program in Goromonzi district, we found that the IPC program failed to achieve any one of the intended outputs. The program was noted to be negatively impacted by a number of factors among them being inadequate resources, inadequate knowledge, inadequate guidelines, high workload and inadequate support and supervision. 
The majority of health facilities were noted to have inadequate inputs and resources which include bins, bin liners, cleaning equipment, PPEs, detergents and enough trained staff. This is a cause of concern because if there is inadequate resources health care workers will adopt the wrong ways of doing things hence exposing themselves and their patients to risk. One component of an effective IPC program is the availability of materials and equipment to perform IPC good practices at the point of care (2). Mushonga et al,(2007) also found the same results and concluded that not using soap during hand washing was attributed to the non-availability of soap at some hand washing points (9).

This lack of resources was aggravated by the fact that there is no a central buying system in the district. Each health facility purchases its own IPC inputs using their own result based funding (RBF) depending on their needs. This poses a challenge since some health care facilities may not prioritise IPC commodities. The price instability also aggravated the problem because it was taking long for the district signatories to authorise requests by rural health facilities to purchase commodities. It was noted that the authorisation could be granted after three days and by then the prices will have changed hence the need to repeat the process again.

There was no prioritisation of the IPC program since none of the visited health facilities in the district had a specific budget for the program. Commodities like HIV test kits and PEP kits which are distributed from a central point (District hospital) were noted to be in adequate supply.

WHO recommends provision of IPC guidance and in-service training of all health care workers at all levels so as to achieve its objectives (2). In this study, the majority of participants had fair IPC knowledge. This was contrary to findings by Mushonga et al who noted that participants had high knowledge levels at Bindura hospital. The level of knowledge could be attributed to lack of trainings in IPC, lack of mentorship and inadequate support and supervision. Although some health care workers received sensitisation and in-service training it was noted not to be enough because those who were training them had not attended the formal training.

Lack of scientific information and the perception that priority is not given to hand hygiene, either on an individual or institutional level is a barrier to good hand hygiene practices (10). This was noted in our study were only $41 \%$ of observed participants wash their hands after patient examination or dressing. Although health care workers had fair knowledge levels this did not reflect in some of their IPC practices in the district. Handwashing facilities had no soap, sun drying of theatre equipment, non-segregation of waste and incorrect sharps material disposal were some of the practices in Goromonzi district. These wrong practices could have been aggravated by lack of IPC guidelines in the district as successful implementation of IPC program depends on the monitoring of adherence with the IPC guideline recommendations. Unavailability of IPC committees also contributed to this since no IPC rounds were being done to identify hazards and make recommendations.

Failure of Goromonzi district to hold IPC meetings and HAI surveillance meetings negatively affected the IPC program performance. This was mainly due to lack of dedicated IPC committees and competing programs in the district. HAI surveillance is the intended outcome of the IPC program but only eleven 
percent of participants were aware of it. Although $69 \%$ of participants knew the ultimate goal of IPC, none of the health facilities in the district had a HAI surveillance system in place. This reflects non prioritisation of the program in the district. In Germany the introduction of a national surveillance programmes with mechanisms for timely feedback was associated with a significant reduction in HAI rates (11). In this study the majority of respondents were aware that they should be screened for TB but only $32 \%$ knew the frequency of screening per year. This could explain why only one facility had waiting time monitoring system at OI/TB clinic and only $33 \%$ with isolation areas for communicable diseases.

Our study had limitation, it was conducted during the indoor residual spaying period hence we only interviewed one EHT therefore insights on environmental control were limited. Information on the availability and adequacy of resources was obtained from interviewees hence possibility of bias. Despite these limitations our study provides insights on the IPC program in the district.

\section{Conclusion}

In conclusion the inputs injected into the IPC program in Goromonzi district were inadequate hence its failure to achieve the intended outputs and outcomes. Inadequate knowledge, lack of mentorship and inadequate support and supervision, unavailability of health worker training plans, absence of IPC committees and unavailability of IPC specific budgets reflected non prioritisation of the program. The IPC practices in Goromonzi district do not reflect the level of knowledge the health workers possess.

We recommended IPC training of all health care workers in the district. The IPC focal person was urged to facilitate urgent establishment of IPC committees and implementation of IPC specific support and supervision with mentorship. As a long term recommendation the DMO should facilitate centralisation of IPC input purchasing in the district.

\section{Declarations}

\section{List of abbreviations}

IPC: Infection, Prevention and Control, HAl: Hospital Acquired Infections, HIV: Human Immunodeficiency Virus, TB: Tuberculosis, WHO: World Health Organisation, AMR: Anti-microbial Resistance, PMD: Provincial Medical Director, DMO: District Medical Officer, $C D C$ : Centres for Disease Control, PEP: Post Exposure Prophylaxis

\section{Ethics approval and consent to participate}

Ethical approval was obtained from the Medical Research Council of Zimbabwe ( MRCZ/ 2018/104). Permission to carry out the study was obtained from the provincial medical director (PMD) Mashonaland East Province, Health Studies Office (HSO) and DMO for Goromonzi district. Written consent was obtained from study participants. Confidentiality was assured and maintained throughout the study by; interviewing each participant privately and ensuring that no information obtained was disclosed to any 
persons other than those relevant for the purposes of the study. No names of participants were written on questionnaires.

\section{Consent for publication}

\section{Not applicable}

\section{Availability of data and material}

The data that support the findings of this study are available from the Ministry of Health \& Child Care Zimbabwe, but restrictions applies to the availability of these data. Data are however available from the authors upon reasonable request and with permission from the Ministry of Health Child Care Zimbabwe.

\section{Competing interests}

The authors declare no competing interests whether financial and non-financial.

\section{Funding}

This study was funded by Mashonaland East Province, Medical Directorate.

\section{Authors' contributions}

EG: conception, design, acquisition, analysis and interpretation of data and drafting the manuscript. STZ: conception, design, acquisition, analysis and interpretation of data and drafting the manuscript. TPJ: conception, design, data analysis, interpretation and reviewing of manuscript draft for important intellectual content. NGT: conception, design, data analysis, interpretation and reviewing of manuscript

draft for important intellectual content. GS: conception, design, data analysis, interpretation and reviewing of manuscript draft for important intellectual content. SC: conception, design, data analysis, interpretation and reviewing of manuscript draft for important intellectual content MT: conception, design, data analysis, interpretation and reviewing of manuscript draft for important intellectual content. All authors have read and approved the final manuscript.

\section{Acknowledgments}

We would like to express our sincere gratitude to the Department of Community Medicine, University of Zimbabwe and Health Studies Office, Zimbabwe for all the help they rendered us during this project. Many thanks go to staff of Goromonzi District who participated in this study. 
Figure 1: The Logic Model

Table 1: Demographic characteristics of health care workers in Goromonzi district, 2018

Table 2: IPC programme resource availability in Goromonzi district health facilities, 2018.

Table 3: Processes for IPC programme in Goromonzi district, 2018

Table 4 Outputs for IPC programme in Goromonzi District, 2018

Table 5: Knowledge of health workers on IPC in Goromonzi district, 2018

Table 6: Reasons for poor performance in IPC, Goromonzi district, 2018.

\section{References}

1. Ministry of Health and Child Care Zimbabwe. National Infection Prevention and Control Policy. 2014.

2. World Health Organization: Regional office for Europe. Guidelines on core components of Infection Prevention and Control programmes at the... national and acute health care facility level. s.l.: World Health Organization; 2016.

3. Ministry of Health and Child Care Z. National Infection Prevention and Control Guidelines. 2013.

4. Ministry of Health and Child Care Z. National Infection Prevention and Control Guidelines. Zimbabwe; 2013.

5. The Direct Medical Costs of Healthcare-Associated Infections in U.S. Hospitals and the Benefits of Prevention.:16.

6. The National Health Strategy for Zimbabwe, 2009-2013. Equity and Quality in Health: A People's Right [Internet]. [cited 2018 Aug 11]. Available from:

http://apps.who.int/medicinedocs/en/d/Js17996en/

7. Situation-Analysis-of-Antimicrobial-use-and-resistance-in-humans-and-animals-in-Zimbabwe-1.pdf [Internet]. [cited 2018 Aug 11]. Available from: https://cddep.org/wpcontent/uploads/2017/10/SITUATION-ANALYSIS-OF-ANTIMICROBIAL-USE-AND-RESISTANCE-INHUMANS-AND-ANIMALS-IN-ZIMBABWE-1.pdf

8. National Health Strategy for Zimbabwe 2016-2020.pdf [Internet]. [cited 2018 Aug 11]. Available from: https://malariaelimination8.org/wpcontent/uploads/2017/02/National\%20Health\%20Strategy\%20for\%20Zimbabwe\%2020162020.pdf

9. Mushonga A. Evaluation of the Infection Control Programme at Bindura Provincial Hospital. 2007.

10. Diwan V, Gustafsson C, Rosales Klintz S, Joshi SC, Joshi R, Sharma M, et al. Understanding Healthcare Workers Self-Reported Practices, Knowledge and Attitude about Hand Hygiene in a Medical Setting in Rural India. PloS One. 2016;11(10):e0163347. 
11. Gastmeier P, Behnke M, Schwab F, Geffers C. Benchmarking of urinary tract infection rates: experiences from the intensive care unit component of the German national nosocomial infections surveillance system. J Hosp Infect. 2011 May 1;78(1):41-4.

\section{Tables}

Table 1: Demographic characteristics of health care workers in Goromonzi district, 2018

\begin{tabular}{llll}
\hline Variable & Characteristic & Frequency n $=71$ & Percentage \\
\hline Sex & Male & 25 & 35.2 \\
& Female & 46 & 64.8 \\
Designation & RGN & 25 & 35.2 \\
& PCN & 15 & 21.2 \\
& Nurse aid & 13 & 18.3 \\
& General hands & 12 & 16.9 \\
& Cook & 3 & 4.2 \\
& Doctor & 2 & 2.8 \\
Median age(years) & EHT & 1 & 1.4 \\
Median years in service & & $38.5\left(\mathrm{Q}_{1}=34, \mathrm{Q}_{3}=44\right)$ & \\
\hline
\end{tabular}

Table 2: IPC program resource availability in Goromonzi district health facilities, 2018. 


\begin{tabular}{llll}
\hline \multicolumn{1}{c}{ Item } & *Targeted facilities & Achieved & Percentage \\
& & $\begin{array}{c}\text { (Facilities with adequate* } \\
\text { Resources) }\end{array}$ & \\
& 15 & 15 & 100 \\
HIV test kits & 15 & 15 & 100 \\
PEP kits & 15 & 14 & 93 \\
Autoclaving equipment & 15 & 13 & 87 \\
Functional toilets & 15 & 12 & 80 \\
Gloves & 15 & 10 & 67 \\
Sharps boxes & 15 & 8 & 53 \\
Brooms/mops & 15 & 6 & 40 \\
National IPC guidelines & 15 & 3 & 20 \\
Bins & 15 & 3 & 20 \\
Bin liners & 15 & 3 & 0 \\
Detergents & 15 & 0 & 20 \\
Budget for IPC & & & \\
\hline
\end{tabular}

*Adequate - No stock outs and 3 months' supply available

$* 15$ = Number of facilities assessed for resources availability

Table 3: Processes for IPC program in Goromonzi district, 2018 


\begin{tabular}{llll}
\hline Process & Target & Achieved & Percentage \\
\hline Training of health workers on IPC & 4 & 0 & 0 \\
$*$ HCW mentorship & 45 & 0 & 0 \\
$*$ Hold IPC meetings & 135 & 0 & 0 \\
$*$ HAI surveillance meetings & 135 & 0 & 100 \\
Support and supervision & 45 & 45 & 100 \\
TB infection control education & Daily & daily & 100 \\
Examination of hospital kitchen food handlers & Once per yearly & once & \\
\hline
\end{tabular}

*HCW mentorship target $=45$ (15 facilities $\mathrm{x}$ once per quarter for 3 quarters)

*IPC and HAI surveillance meetings target $=135$ (15 facilities $\mathrm{x}$ once per month $\mathrm{x} 9$ months)

Table 4: Outputs for IPC program in Goromonzi District, 2018 


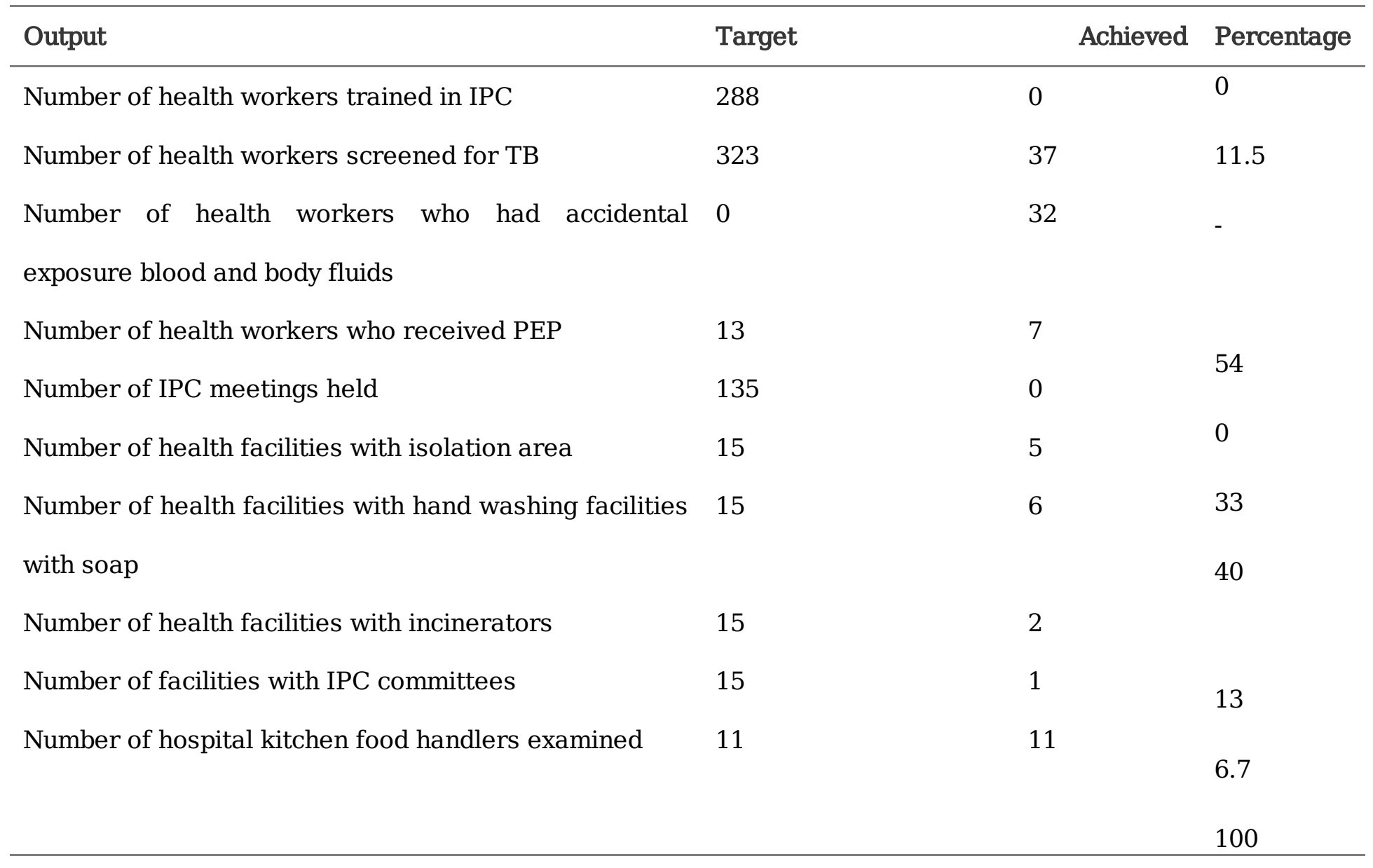

Table 5: Knowledge of health workers on IPC in Goromonzi district, 2018 


\begin{tabular}{lll}
\hline Attribute & Frequency n=71 & Percentage \\
\hline Have heard of IPC & 67 & 94.4 \\
Know the ultimate goal of IPC & 49 & 69.0 \\
Know at least 3 standard precautions of IPC & 34 & 47.9 \\
Know how to dilute disinfectants & 36 & 50.7 \\
Know how many times are they supposed & 23 & 32.4 \\
to be screened for TB & & 24.0 \\
Know bin colour coding for waste segregation & 17 & 11.3 \\
Aware of HAI surveillance & 8 & \\
\hline
\end{tabular}

Table 6: Reasons for poor performance in IPC, Goromonzi district, 2018.

\begin{tabular}{|c|c|c|c|}
\hline \multirow{2}{*}{$\begin{array}{l}\text { Reason } \\
\text { Inadequate resources }\end{array}$} & \multicolumn{2}{|c|}{ Frequency $n=71$} & \multirow[t]{2}{*}{ Percentage } \\
\hline & 64 & 90.1 & \\
\hline Inadequate knowledge & 46 & 64.8 & \\
\hline Inadequate support \& supervision & 43 & 60.6 & \\
\hline High workload & 43 & 60.6 & \\
\hline No mentorship & 41 & & \\
\hline \multirow[t]{2}{*}{ Lack of guidelines } & 36 & 57.8 & \\
\hline & & 50.7 & \\
\hline
\end{tabular}

Figures 


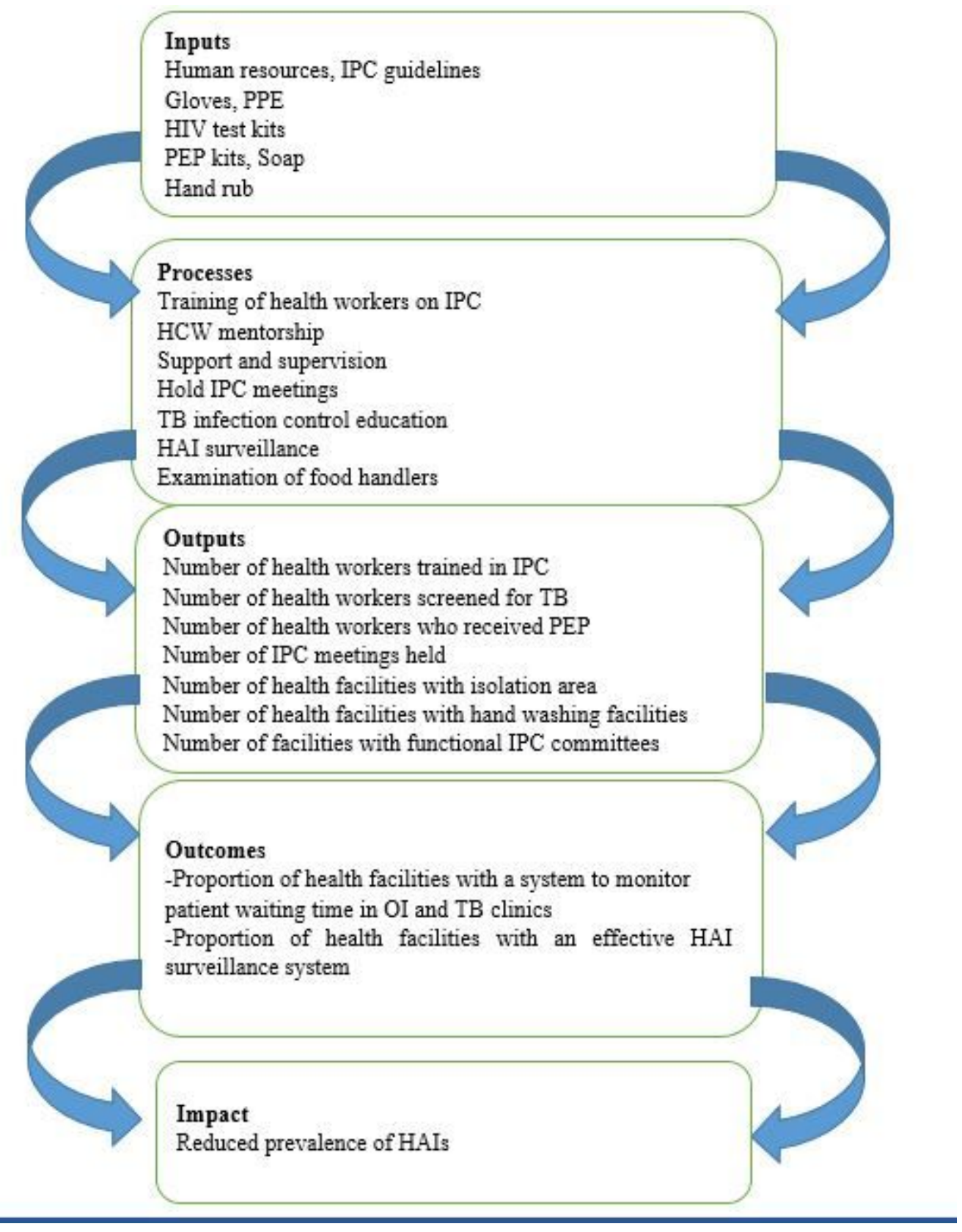

Figure 1

The Logic Model

\section{Supplementary Files}

This is a list of supplementary files associated with this preprint. Click to download.

- additionalFileQuestinnaire.docx 